\title{
Relaying for Multiple Sources in the Absence of Codebook Information
}

\author{
Ye Tian and Aylin Yener \\ Wireless Communications and Networking Laboratory \\ Electrical Engineering Department \\ The Pennsylvania State University, University Park, PA 16802 \\ yetian@psu.eduｙener@ee.psu.edu
}

\begin{abstract}
This work investigates the optimality of compressand-forward (CF) type relaying in wireless networks when the relay does not know the codebooks used by the sources. The relay is called oblivious for this reason. The relay-destination links are assumed to be out-of-band with finite capacity. We study two multi-source relay networks, namely the multiple access relay channel (MARC) and the interference relay channel (IFRC), both with an oblivious relay. For the MARC with an oblivious relay, we derive a new outerbound and show that the capacity region can be established using a generalized CF (GCF) scheme, where the destinations jointly decode the compression indices and the source messages. In particular, we observe that, for multi-source relay networks, GCF scheme achieves higher individual rates than the conventional CF scheme with sequential decoding of compression indices and source messages. For the IFRC with an oblivious relay, we focus on the case where the destinations know all the codebooks used by the sources. We establish a new strong interference condition, under which we derive a new outerbound and show that it is achievable using GCF scheme, thus establishing the capacity region of IFRC with an oblivious relay.
\end{abstract}

\section{INTRODUCTION}

Relaying is a fundamental operation in a wireless network. The simplest channel that models this operation is the classical relay channel with one source, one destination and one relay, in the context of which various relaying strategies have been proposed [1], [2]. The capacity of the relay channel is known for special cases, e.g. [1] and [2], where the optimality of decodeand-forward (DF), hash-and-forward (HF) and compress-andforward (CF) is established respectively. For multi-user relay networks, the multiple access relay channel (MARC) and interference relay channel (IFRC) have been studied in [3][6]. The capacity for MARC and IFRC are also only known under special conditions using DF [3], [5].

A default assumption for analysis of the models above is that all codebooks used by the sources are known at the relay. In future wireless networks, the wireless devices for different applications may co-exist in the same area sharing the same resources, and the mobility can cause frequent changes in the distribution of the wireless devices. Exchanging codebook information could then lead to excessive overhead. It is thus interesting to investigate the fundamental performance limits of a network when the relay nodes do not have the codebook information.
To model the uncertainty about the codebook information at the relay, i.e., an oblivious relay, reference [7] has proposed a model which uses randomized encoding at the source, and the source shares some common randomness about the encoding function, i.e., the codebook information, with the destination. We can model the uncertainty about the codebook information at the relay by not informing relay the common randomness of the codebook. This idea is further developed in [8], which has established the capacity of the primitive relay channel with an oblivious relay using CF relaying, where the term primitive refers to the relay-destination links being out-of-band and of finite-capacity [2].

When the uncertainty of codebook information is incorporated in the model, the decoding operation of IFRC is further classified as interference-aware decoding and interferenceoblivious decoding in reference [8]. For the primitive IFRC (PIFRC) with interference-oblivious decoding, reference [8] established the capacity region using $\mathrm{CF}$ relaying and treating interference as noise at the destinations. This work also investigated the PIFRC with interference-aware decoding, and the sum capacity is established using CF relaying when the destinations have the same statistics in their received signals, which reduces the channel to primitive MARC (PMARC) with an oblivious relay. However, the optimality of CF scheme with respect to the individual rates for PMARC with an oblivious relay is still unknown. In addition, the capacity region of the general PIFRC with an oblivious relay under interferenceaware decoding is unknown.

In this paper, we make progress towards understanding the optimal relaying strategies under oblivious relaying framework. Specifically, we establish the optimality of a generalized $\mathrm{CF}$ (GCF) scheme, where the destinations jointly decode the compression index and source messages, in contrast with the $\mathrm{CF}$ scheme, where the destinations decode compression index and source messages in a sequential manner. For the PMARC with an oblivious relay, we derive new outerbounds and show that GCF indeed achieves the entire capacity region. We also show that the GCF can outperform CF in terms of individual rate. We then focus on the PIFRC with an oblivious relay under interference-aware decoding. We demonstrate a new strong interference condition, under which we derive new outerbounds and show that the capacity region of the PIFRC 
with an oblivious relay can be established using GCF.

\section{System Model}

We consider a general primitive multiuser network with $M$ sources and $K$ destinations assisted by an oblivious relay. The model is then specified into MARC and IFRC in the subsequent sections. We define $\mathcal{M}=\{1,2, \cdots, M\}$ as the index set of the sources and $\mathcal{K}=\{1,2, \cdots, K\}$ as the index set of the destinations. We define the message set at source $i$ as $\mathcal{W}_{i}=\left\{1,2, \cdots, 2^{n R_{i}}\right\}$, and the alphabet for source $i$ as $\mathcal{X}_{i}$. We also define the set of codebooks at source $i$ as all possible combinations of length $n$ codewords for each message, where the codewords consist of symbols chosen from the alphabet. The number of codebooks is thus $\left|\mathcal{X}_{i}\right|^{n 2^{n R_{i}}}$ for source $i$. We define an index set of all the codebooks for source $i$ as $\mathcal{F}_{i}=\left\{1,2, \cdots,\left|\mathcal{X}_{i}\right|^{n 2^{n R_{i}}}\right\}$.

Definition of the source encoder: We follow the definition in [8] to allow time sharing. We define a $\left(n, R_{1}, R_{2}, \cdots, R_{M}\right)$ code for the $M$-source $K$-destination channel assisted by an oblivious relay with time-sharing as $\left(P_{F_{i} \mid Q^{n}}, \phi_{i}^{n}\right)(i \in \mathcal{M})$, where $P_{F_{i} \mid Q^{n}}\left(f_{i} \mid q^{n}\right)$ is the probability of choosing the codebook $f_{i} \in \mathcal{F}_{i}$ conditioned on the time sharing sequence $q^{n} \in$ $\mathcal{Q}^{n} . \phi_{i}^{n}$ is the encoding function such that $x_{i}^{n}=\phi_{i}^{n}\left(w_{i}, f_{i}\right)$, where $w_{i} \in \mathcal{W}_{i}$. The probability of selecting the codebook $f_{i}$ for source $i$ conditioned on $q^{n}$ is

$$
P_{F_{i} \mid Q^{n}}\left(f_{i} \mid q^{n}\right)=\prod_{w_{i}=1}^{2^{n R_{i}}} P_{X_{i}^{n} \mid Q^{n}}\left(\phi_{i}^{n}\left(w_{i}, f_{i}\right) \mid q^{n}\right)
$$

where $P_{X_{i}^{n} \mid Q^{n}}\left(x_{i}^{n} \mid q^{n}\right)=\prod_{t=1}^{n} P_{X_{i} \mid Q}\left(x_{i, t} \mid q_{t}\right)$.

Note that the codebook and message are selected independently, i.e.,

$$
P_{F_{i} W_{i} \mid Q^{n}}\left(f_{i}, w_{i} \mid q^{n}\right)=P_{F_{i} \mid Q^{n}}\left(f_{i} \mid q^{n}\right) \cdot 2^{-n R_{i}} .
$$

Based on this formulation, we have

$$
\begin{aligned}
& P_{X_{i}^{n} \mid Q^{n}}\left(x_{i}^{n} \mid q^{n}\right)=\prod_{j=1}^{n} P_{X_{i} \mid Q}\left(x_{i, j} \mid q_{j}\right), \\
& P_{Y_{i}^{n} \mid Q^{n}}\left(y_{i}^{n} \mid q^{n}\right)=\prod_{j=1}^{n} P_{Y_{i} \mid Q}\left(y_{i, j} \mid q_{j}\right),
\end{aligned}
$$

i.e., without the codebook information, the destination sees the transmitted sequence from the source and its received sequence as generated independently. This relation can be derived following Lemma 1 in [7]. Note that the randomized selection of the codebook is only to model the uncertainty of the codebook at oblivious nodes, and it does not represent the actual communication scenario. More detailed explanation on the system model can be found in [7] and Remark 2-4 in [8].

Definition of the channel: The channel is discrete memoryless, and consists of $M$ input alphabets and $K+1$ output alphabets, a channel transition probability, and $K$ out-of-band finite capacity links from the relay to the destinations, i.e.,

$$
\mathcal{X}_{\mathcal{M}}, p\left(y_{1} y_{2} \cdots y_{K} y_{R} \mid x_{1} x_{2} \cdots x_{M}\right), \mathcal{Y}_{\mathcal{K}}, \mathcal{Y}_{R}, C_{\mathcal{K}}
$$

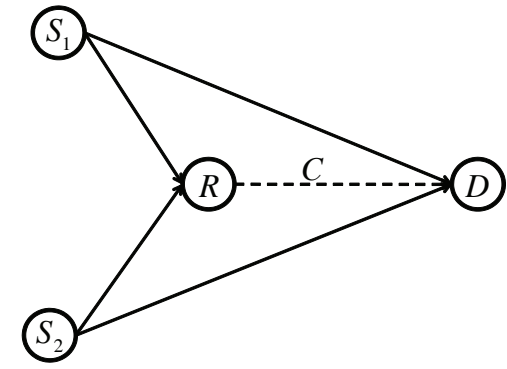

Fig. 1. Primitive multiple access relay channel with an oblivious relay.

Definition of the relay encoder: The relay does not know the codebooks used by the sources. It communicates to each destination with an out-of-band finite capacity link. The messages are generated according to an encoding function

$$
\phi_{R}^{n}: \mathcal{Y}_{R}^{n} \times \mathcal{Q}^{n} \rightarrow \mathcal{S}_{1} \times \mathcal{S}_{2} \cdots \mathcal{S}_{K}
$$

with $\mathcal{S}_{k}=\left\{1,2, \cdots, 2^{n C_{k}}\right\}, k \in \mathcal{K}$. We denote $\left(S_{1}, S_{2}, \cdots, S_{K}\right)=\phi_{R}^{n}\left(Y_{R}^{n} \mid q^{n}\right)$ as the messages generated by the relay.

Definition of the decoder: We assume that the destinations know all the codebooks used by the sources. We consider both multicast (MC) and unicast (UC) transmissions. For multicast transmission, i.e., each source wishes to transmit a message to all destinations, we define the decoding function at destination $j \in \mathcal{K}$ as

$g_{j}^{M C}: \mathcal{Q}^{n} \times \mathcal{F}_{1} \times \mathcal{F}_{2} \cdots \mathcal{F}_{M} \times \mathcal{S}_{j} \times \mathcal{Y}_{j}^{n} \rightarrow \mathcal{W}_{1} \times \mathcal{W}_{2} \cdots \mathcal{W}_{M}$.

A set of rates $\left(R_{1}, R_{2}, \cdots, R_{M}\right)$ is achievable if there exists $\left(P_{F_{i} \mid Q^{n}}, \phi_{i}^{n}\right)$ for all $i \in \mathcal{K}$ such that $\left(\hat{W}_{1}^{i}, \cdots, \hat{W}_{M}^{i}\right)=$ $g_{i}^{M C}\left(Q^{n}, F_{1}, \cdots, F_{M}, S_{i}, Y_{i}^{n}\right)$, and $\operatorname{Pr}\left\{\cup_{i=1}^{M} \cup_{j=1}^{K} \hat{W}_{i}^{j} \neq\right.$ $\left.W_{i}\right\} \rightarrow 0$ as $n \rightarrow \infty$.

For unicast transmission, we assume that $\mathcal{M}=\mathcal{K}$, i.e., each source only wishes to transmit a message to its intended destination, we define the decoding function at destination $j \in$ $\mathcal{M}$ as

$$
g_{j}^{U C}: \mathcal{Q}^{n} \times \mathcal{F}_{1} \times \mathcal{F}_{2} \cdots \mathcal{F}_{M} \times \mathcal{S}_{j} \times \mathcal{Y}_{j}^{n} \rightarrow \mathcal{W}_{j}
$$

A set of rates $\left(R_{1}, R_{2}, \cdots, R_{M}\right)$ is achievable if there exists $\left(P_{F_{i} \mid Q^{n}}, \phi_{i}^{n}\right)$ for all $i \in \mathcal{K}$ such that $\hat{W}_{i}=$ $g_{i}^{U C}\left(Q^{n}, F_{1}, \cdots, F_{M}, S_{i}, Y_{i}^{n}\right)$, and $\operatorname{Pr}\left\{\cup_{i=1}^{M} \hat{W}_{i} \neq W_{i}\right\} \rightarrow 0$ as $n \rightarrow \infty$.

\section{CApacity Region for the Multiple AcCess Channel With an OBlivious Relay}

In this section, we study the primitive multiple access channel (PMARC) with an oblivious relay, i.e., $M=2, K=1$ for multicast transmission, which is shown in Figure 1. The relay-destination link is out-of-band with finite capacity $C$, and we denote the message transmitted from relay to the destination as $S$. 


\section{A. Main Result}

Theorem 1: The following rate region is the capacity region of PMARC with oblivious relaying:

$$
\begin{aligned}
& R_{1}<I\left(X_{1} ; \hat{Y}_{R} Y \mid X_{2} Q\right) \\
& R_{1}<I\left(X_{1} ; Y \mid X_{2} Q\right)+C-I\left(Y_{R} ; \hat{Y}_{R} \mid X_{1} X_{2} Y Q\right) \\
& R_{2}<I\left(X_{2} ; \hat{Y}_{R} Y \mid X_{1} Q\right) \\
& R_{2}<I\left(X_{2} ; Y \mid X_{1} Q\right)+C-I\left(Y_{R} ; \hat{Y}_{R} \mid X_{1} X_{2} Y Q\right) \\
& R_{1}+R_{2}<I\left(X_{1} X_{2} ; \hat{Y}_{R} Y \mid Q\right) \\
& R_{1}+R_{2}<I\left(X_{1} X_{2} ; Y \mid Q\right)+C-I\left(Y_{R} ; \hat{Y}_{R} \mid X_{1} X_{2} Y Q\right)
\end{aligned}
$$

for all distributions

$$
p(q) p\left(x_{1} \mid q\right) p\left(x_{2} \mid q\right) p\left(\hat{y}_{R} \mid y_{R} q\right)
$$

Proof: The achievability can be proved by using the GCF relaying, which is first proposed in [9] for the relay channel and then generalized to multiuser networks in [6]. The detailed scheme for PMARC can be found in [10]. For outerbounds, we need to utilize the property (3) and appropriately define the random variable $\hat{Y}_{R}$. To illustrate the proof approach with clarity, we only provide the proof of the individual rates. The sum rate upperbounds can be derived in the same fashion and thus are omitted. For details, see [10]. For the individual rate $R_{1}$, we have

$$
\begin{aligned}
n R_{1} & =H\left(W_{1}\right) \\
& =H\left(W_{1} \mid Q^{\prime}\right) \\
& \leq I\left(W_{1} ; Y^{n} S F_{1} F_{2} \mid Q^{\prime} W_{2}\right)+n \epsilon_{n} \\
& =I\left(W_{1} ; F_{1} F_{2} \mid Q^{\prime} W_{2}\right)+I\left(W_{1} ; Y^{n} S \mid Q^{\prime} W_{2} F_{2} F_{1}\right) \\
& \leq I\left(F_{1} W_{1} ; Y^{n} S \mid Q^{\prime} W_{2} F_{2}\right)+n \epsilon_{n} \\
& \leq I\left(X_{1}^{n} ; Y^{n} S \mid Q^{\prime} X_{2}^{n}\right)+n \epsilon_{n}
\end{aligned}
$$

where $Q^{\prime}=Q^{n}, \epsilon_{n} \rightarrow 0$ as $n \rightarrow \infty$ and (19) follows from the independence between $W_{1}$ and $F_{1} F_{2}$. Due to properties (3) and (4), we have that the symbols from source sequences are independent for each instance $i$ conditioned on $Q^{\prime}$ without conditioning on the codebook information. When we combine this property and the memoryless property of the channel, together with the definition of relay encoder, we have the Markov chain between the random variables illustrated in Figure 2.

We can further bound (21) with two different methods.

$$
\begin{aligned}
& I\left(X_{1}^{n} ; Y^{n} S \mid Q^{\prime} X_{2}^{n}\right) \\
& =H\left(X_{1}^{n} \mid Q^{\prime} X_{2}^{n}\right)-H\left(X_{1}^{n} \mid Y^{n} S Q^{\prime} X_{2}^{n}\right) \\
& =\sum_{i=1}^{n} H\left(X_{1 i} \mid X_{2 i} Q^{\prime}\right)-\sum_{i=1}^{n} H\left(X_{1 i} \mid Y^{n} S Q^{\prime} X_{2}^{n} X_{1}^{i-1}\right) \\
& \leq \sum_{i=1}^{n} H\left(X_{1 i} \mid X_{2 i} Q^{\prime}\right)-\sum_{i=1}^{n} H\left(X_{1 i} \mid \hat{Y}_{R i} X_{2 i} Y_{i} Q^{\prime}\right) \\
& =\sum_{i=1}^{n} I\left(X_{1 i} ; \hat{Y}_{R i} Y_{i} \mid X_{2 i} Q^{\prime}\right)
\end{aligned}
$$

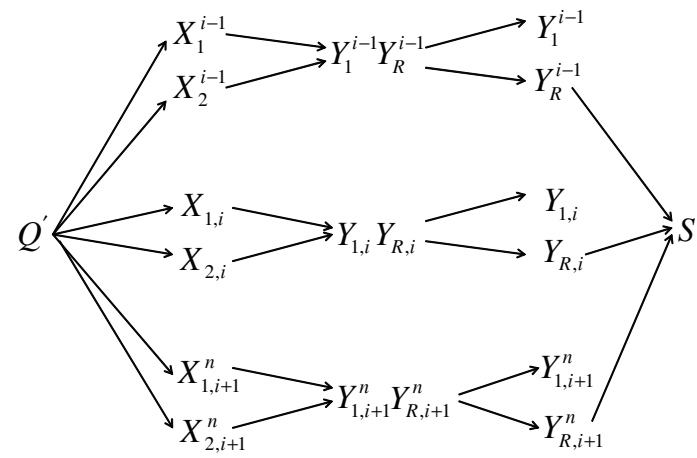

Fig. 2. Markov chain between random variables.

where (24) follows from the independence between source inputs and the property (3), and in (25), since conditioning reduces entropy, we add some random variables in the condition of the second term to form $\hat{Y}_{R i}$, which is defined as

$$
\hat{Y}_{R i}=S X_{1}^{i-1} X_{1, i+1}^{n} X_{2}^{i-1} X_{2, i+1}^{n} Y^{i-1} Y_{i+1}^{n} Y_{R}^{i-1} .
$$

We can also bound the term (21) in the following way:

$$
\begin{aligned}
I & \left(X_{1}^{n} ; Y^{n} S \mid Q^{\prime} X_{2}^{n}\right) \\
= & I\left(X_{1}^{n} ; Y^{n} \mid Q^{\prime} X_{2}^{n}\right)+I\left(X_{1}^{n} ; S \mid Q^{\prime} X_{2}^{n} Y^{n}\right) \\
= & H\left(Y^{n} \mid Q^{\prime} X_{2}^{n}\right)-H\left(Y^{n} \mid Q^{\prime} X_{2}^{n} X_{1}^{n}\right)+H\left(S \mid Q^{\prime} X_{2}^{n} Y^{n}\right) \\
& -H\left(S \mid Q^{\prime} X_{1}^{n} X_{2}^{n} Y^{n}\right) \\
\leq & \sum_{i=1}^{n} H\left(Y_{i} \mid X_{2 i} Q^{\prime}\right)-\sum_{i=1}^{n} H\left(Y_{i} \mid X_{1 i} X_{2 i} Q^{\prime}\right)+H(S) \\
& -\left(H\left(S \mid Q^{\prime} X_{1}^{n} X_{2}^{n} Y^{n}\right)-H\left(S \mid Q^{\prime} X_{1}^{n} X_{2}^{n} Y^{n} Y_{R}^{n}\right)\right) \\
\leq & \sum_{i=1}^{n} I\left(X_{1 i} ; Y_{i} \mid X_{2 i} Q^{\prime}\right)+n C-I\left(S ; Y_{R}^{n} \mid Q^{\prime} X_{1}^{n} X_{2}^{n} Y^{n}\right) \\
= & \sum_{i=1}^{n} I\left(X_{1 i} ; Y_{i} \mid X_{2 i} Q^{\prime}\right)+n C \\
& -\sum_{i=1}^{n} I\left(S ; Y_{R i} \mid Q^{\prime} X_{1}^{n} X_{2}^{n} Y^{n} Y_{R}^{i-1}\right) \\
= & \sum_{i=1}^{n} I\left(X_{1 i} ; Y_{i} \mid X_{2 i} Q^{\prime}\right)-\sum_{i=1}^{n}\left(H\left(Y_{R i} \mid Q^{\prime} X_{1}^{n} X_{2}^{n} Y^{n} Y_{R}^{i-1}\right)\right. \\
& \left.-H\left(Y_{R i} \mid S Q^{\prime} X_{1}^{n} X_{2}^{n} Y^{n} Y_{R}^{i-1}\right)\right)+n C \\
= & \sum_{i=1}^{n} I\left(X_{1 i} ; Y_{i} \mid X_{2 i} Q^{\prime}\right)+n C-\sum_{i=1}^{n}\left(H\left(Y_{R i} \mid Q^{\prime} X_{1 i} X_{2 i} Y_{i}\right)\right. \\
& \left.-H\left(Y_{R i} \mid \hat{Y}_{R i} X_{1 i} X_{2 i} Y_{i} Q^{\prime}\right)\right) \\
& \sum_{i=1}^{n} I\left(X_{1 i} ; Y_{i} \mid X_{2 i} Q^{\prime}\right)+n C-\sum_{i=1}^{n} I\left(\hat{Y}_{R i} ; Y_{R i} \mid X_{1 i} X_{2 i} Y_{i} Q^{\prime}\right) \\
& -n 3) \\
&
\end{aligned}
$$

where (34) follows from the Markov chain in Figure 2 and the way we define the random variable $\hat{Y}_{R i}$. The result can be obtained by introducing another time sharing random variable 
$Q^{\prime \prime} \sim \mathcal{U}(\{1,2, \cdots, n\})$ and setting $Q=\left(Q^{\prime \prime}, Q^{\prime}\right)$. The way we define the random variable $\hat{Y}_{R i}$ implies the distribution (15). The individual rate $R_{2}$ can be obtained in a similar fashion.

Remark 1: The result can be readily extended to $M$-user MARC with an oblivious relay, or a multicast network with $M$ sources and $K$ destinations.

\section{B. Comparison between $G C F$ and $C F$ relaying}

It is shown that GCF and CF achieve the same rate for single source single destination channel with relays [9], [11]. For channels with multiple destination, it is shown that GCF outperforms $\mathrm{CF}$ in [6]. For channels with two sources and one destination, i.e., the MARC, it is interesting to further investigate the performance between the two schemes. Note that in the following analysis, we focus on the model where the relay-destination link is out-of-band with finite capacity for the clarity of illustration. The discussion on the achievable rate region using GCF and $\mathrm{CF}$ holds regardless the relay is oblivious or not.

The achievable rate region for PMARC due to the $\mathrm{CF}$ strategy can be obtained as follows:

$$
\begin{aligned}
& R_{1}<I\left(X_{1} ; \hat{Y}_{R} Y \mid X_{2} Q\right) \\
& R_{2}<I\left(X_{2} ; \hat{Y}_{R} Y \mid X_{1} Q\right) \\
& R_{1}+R_{2}<I\left(X_{1} X_{2} ; \hat{Y}_{R} Y \mid Q\right)
\end{aligned}
$$

with

$$
C \geq I\left(Y_{R} ; \hat{Y}_{R} \mid Y Q\right)
$$

for all distributions

$$
p(q) p\left(x_{1} \mid q\right) p\left(x_{2} \mid q\right) p\left(\hat{y}_{R} \mid y_{R} q\right) .
$$

We can see that the maximization of the rates also depends on the random variable $\hat{Y}_{R}$ satisfying (38). It is easy to see that the maximum achievable individual rate for one user, i.e., the rate point $\left(R_{1}, 0\right)$ or $\left(0, R_{2}\right)$ and the maximum achievable sum rate are the same for the two schemes, following an argument similar to the one used in [9]. For the other rate points on the border of the rate region, say the maximum of rate $R_{1}$ for some fixed $R_{2}$, the rate due to GCF is potentially larger than that of $\mathrm{CF}$. To see this, the maximum of rate (35) is obtained with respect to all distribution $p(q) p\left(x_{1} \mid q\right) p\left(x_{2} \mid q\right) p\left(\hat{y}_{R} \mid y_{R} q\right)$ such that (38) is satisfied. For the rate of GCF, which is $\min ((9),(10))$, we first constrain the set of input distribution to the set which guarantees that (9) is the minimum of the two individual rates. This constraint on input distribution only reduces the maximum rate $R_{1}$ of the GCF scheme. The maximum of (9) is then obtained with respect to the distribution $p(q) p\left(x_{1} \mid q\right) p\left(x_{2} \mid q\right) p\left(\hat{y}_{R} \mid y_{R} q\right)$ such that $C \geq I\left(Y_{R} ; \hat{Y}_{R} \mid Y X_{2} Q\right)$. It is easy to see that $I\left(Y_{R} ; \hat{Y}_{R} \mid Y Q\right) \geq I\left(Y_{R} ; \hat{Y}_{R} \mid Y X_{2} Q\right)$. Thus the maximization of (9) is potentially larger than (35) since the distribution can be chosen from a larger set. The achievable rate region using $\mathrm{GCF}$ is thus potentially larger than that of $\mathrm{CF}$.

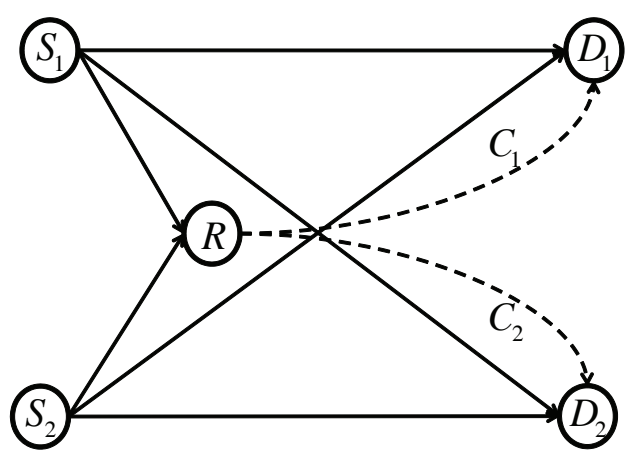

Fig. 3. Primitive interference relay channel with an oblivious relay.

\section{CApacity Region for The Primitive \\ INTERFERENCE RELAY CHANNEL WITH AN OBLIVIOUS RELAY}

In this section, we present a result for the primitive interference relay channel (PIFRC) with an oblivious relay, i.e., $M=2, K=2$ for unicast transmission. We assume that the relay connects to destination 1 (2) with an out-of-band link with capacity $C_{1}\left(C_{2}\right)$ and the destinations are assumed to be interference-aware, which is shown in Figure 3.

Theorem 2: The following rate region is the capacity region for the PIFRC with an oblivious relay:

$$
\begin{aligned}
& R_{1}<I\left(X_{1} ; \hat{Y}_{R 1} Y_{1} \mid X_{2} Q\right) \\
& R_{1}<I\left(X_{1} ; Y_{1} \mid X_{2} Q\right)+C_{1}-I\left(Y_{R} ; \hat{Y}_{R 1} \mid X_{1} X_{2} Y_{1} Q\right) \\
& R_{2}<I\left(X_{2} ; \hat{Y}_{R 2} Y_{2} \mid X_{1} Q\right) \\
& R_{2}<I\left(X_{2} ; Y_{2} \mid X_{1} Q\right)+C_{2}-I\left(Y_{R} ; \hat{Y}_{R 2} \mid X_{1} X_{2} Y_{2} Q\right) \\
& R_{1}+R_{2}<I\left(X_{1} X_{2} ; \hat{Y}_{R 1} Y_{1} \mid Q\right) \\
& R_{1}+R_{2}<I\left(X_{1} X_{2} ; Y_{1} \mid Q\right)+C_{1}-I\left(Y_{R} ; \hat{Y}_{R 1} \mid X_{1} X_{2} Y_{1} Q\right) \\
& R_{1}+R_{2}<I\left(X_{1} X_{2} ; \hat{Y}_{R 2} Y_{2} \mid Q\right) \\
& R_{1}+R_{2}<I\left(X_{1} X_{2} ; Y_{2} \mid Q\right)+C_{2}-I\left(Y_{R} ; \hat{Y}_{R 2} \mid X_{1} X_{2} Y_{2} Q\right)
\end{aligned}
$$

under the condition that the channel transition probability prompts the following strong interference conditions

$$
\begin{aligned}
& I\left(X_{1} ; Y_{1} Y_{R} \mid X_{2}\right) \leq I\left(X_{1} ; Y_{2} \mid X_{2}\right) \\
& I\left(X_{2} ; Y_{2} Y_{R} \mid X_{1}\right) \leq I\left(X_{2} ; Y_{1} \mid X_{1}\right)
\end{aligned}
$$

for all distributions

$$
p(q) p\left(x_{1} \mid q\right) p\left(x_{2} \mid q\right) p\left(\hat{y}_{R 1} \mid y_{R} q\right) p\left(\hat{y}_{R 2} \mid y_{R} q\right)
$$

Proof: The achievability follows from the GCF relaying, which is similar to the one we used for Theorem 1. For the outerbounds, we need to utilize the strong interference condition (48) and (49). In fact, the conditions (48) and (49) implies that (see [12] for details)

$$
I\left(X_{1}^{n} ; Y_{1}^{n} Y_{R}^{n} \mid X_{2}^{n} Q\right) \leq I\left(X_{1}^{n} ; Y_{2}^{n} \mid X_{2}^{n} Q\right)
$$




$$
I\left(X_{2}^{n} ; Y_{2}^{n} Y_{R}^{n} \mid X_{1}^{n} Q\right) \leq I\left(X_{2}^{n} ; Y_{1}^{n} \mid X_{1}^{n} Q\right)
$$

Since $S_{1}$ and $S_{2}$ are functions of $Y_{R}^{n}$, we have

$$
\begin{gathered}
I\left(X_{1}^{n} ; Y_{1}^{n} S_{1} \mid X_{2}^{n} Q\right) \leq I\left(X_{1}^{n} ; Y_{1}^{n} Y_{R}^{n} \mid X_{2}^{n} Q\right) \\
\quad \leq I\left(X_{1}^{n} ; Y_{2}^{n} \mid X_{2}^{n} Q\right) \leq I\left(X_{1}^{n} ; Y_{2}^{n} S_{2} \mid X_{2}^{n} Q\right) \\
I\left(X_{2}^{n} ; Y_{2}^{n} S_{2} \mid X_{1}^{n} Q\right) \leq I\left(X_{2}^{n} ; Y_{2}^{n} Y_{R}^{n} \mid X_{1}^{n} Q\right) \\
\quad \leq I\left(X_{2}^{n} ; Y_{1}^{n} \mid X_{1}^{n} Q\right) \leq I\left(X_{2}^{n} ; Y_{1}^{n} S_{1} \mid X_{1}^{n} Q\right)
\end{gathered}
$$

due to the fact that $\left(S_{1}, S_{2}\right)=f\left(Y_{R}^{n}\right)$

The outerbounds for individual rates can be obtained by setting

$$
\begin{aligned}
& \hat{Y}_{R 1, i}=S_{1} X_{1}^{i-1} X_{1, i+1}^{n} X_{2}^{i-1} X_{2, i+1}^{n} Y_{1}^{i-1} Y_{1, i+1}^{n} Y_{R}^{i-1}, \\
& \hat{Y}_{R 2, i}=S_{2} X_{1}^{i-1} X_{1, i+1}^{n} X_{2}^{i-1} X_{2, i+1}^{n} Y_{2}^{i-1} Y_{2, i+1}^{n} Y_{R}^{i-1} .
\end{aligned}
$$

using similar steps as in Theorem 1.

For the sum rate outerbounds, we have

$$
\begin{aligned}
n & \left(R_{1}+R_{2}\right) \\
= & H\left(W_{1}\right)+H\left(W_{2}\right) \\
= & H\left(W_{1} \mid Q\right)+H\left(W_{2} \mid Q\right) \\
\leq & I\left(W_{1} ; Y_{1}^{n} S_{1} F_{1} F_{2} \mid W_{2} Q\right)+I\left(W_{2} ; Y_{2}^{n} S_{2} F_{1} F_{2} \mid Q\right)+n \epsilon_{n} \\
= & I\left(W_{1} ; F_{1} F_{2} \mid Q W_{2}\right)+I\left(W_{1} ; Y_{1}^{n} S_{1} \mid Q W_{2} F_{1} F_{2}\right) \\
& +I\left(W_{2} ; F_{1} F_{2} \mid Q\right)+I\left(W_{2} ; Y_{2}^{n} S_{2} \mid Q F_{1} F_{2}\right)+n \epsilon_{n} \\
\leq & I\left(W_{1} ; Y_{1}^{n} S_{1} \mid Q F_{2} W_{2} F_{1}\right)+I\left(W_{2} F_{2} ; Y_{2}^{n} S_{2} \mid Q F_{1}\right)+n \epsilon_{n} \\
\leq & I\left(X_{1}^{n} ; Y_{1}^{n} S_{1} \mid Q X_{2}^{n} F_{1}\right)+I\left(X_{2}^{n} ; Y_{2}^{n} S_{2} \mid Q F_{1}\right)+n \epsilon_{n} \\
\leq & I\left(X_{1}^{n} ; Y_{2}^{n} S_{2} \mid Q X_{2}^{n} F_{1}\right)+I\left(X_{2}^{n} ; Y_{2}^{n} S_{2} \mid Q F_{1}\right)+n \epsilon_{n} \\
= & I\left(X_{1}^{n} X_{2}^{n} ; Y_{2}^{n} S_{2} \mid Q\right)
\end{aligned}
$$

From this we can derive the bounds (46) and (47) following from similar steps as in Theorem 1, and the bounds (44) and (45) can be obtained using condition (49).

With the auxiliary random variables specified by (55) and (56), the probability distribution is factorized as

$$
p(q) p\left(x_{1} \mid q\right) p\left(x_{2} \mid q\right) p\left(\hat{y}_{R 1} \hat{y}_{R 2} \mid y_{R} q\right) p\left(y_{1} y_{2} y_{R} \mid x_{1} x_{2}\right)
$$

Note that the input distribution

$$
p(q) p\left(x_{1} \mid q\right) p\left(x_{2} \mid q\right) p\left(\hat{y}_{R 1} \mid y_{R} q\right) p\left(\hat{y}_{R 2} \mid y_{R} q\right) p\left(y_{1} y_{2} y_{R} \mid x_{1} x_{2}\right)
$$

yields the same rate region as the one specified by (63). This is because all the rates only depend on the marginal distribution

$$
\begin{aligned}
& p(q) p\left(x_{1} \mid q\right) p\left(x_{2} \mid q\right) p\left(\hat{y}_{R 1} \mid y_{R} q\right) p\left(y_{1} y_{R} \mid x_{1} x_{2}\right), \\
& p(q) p\left(x_{1} \mid q\right) p\left(x_{2} \mid q\right) p\left(\hat{y}_{R 2} \mid y_{R} q\right) p\left(y_{2} y_{R} \mid x_{1} x_{2}\right) .
\end{aligned}
$$

For each distribution factorized as (63), we can always find a distribution factorized as (64) that yields the same marginal distribution (65) and (66).

It then suffices to constrain the probability distribution to the form of (63).

Discussion on the strong interference condition: To get a more intuitive understanding of the strong interference conditions, we further investigate the Gaussian channel

$$
Y_{1}=h_{11} X_{1}+h_{21} X_{2}+Z_{1}
$$

$$
\begin{gathered}
Y_{2}=h_{12} X_{1}+h_{22} X_{2}+Z_{2} \\
Y_{R}=h_{1 R} X_{1}+h_{2 R} X_{2}+Z_{R},
\end{gathered}
$$

where $Z_{1}, Z_{2}, Z_{R} \sim \mathcal{N}(0,1)$.

It can be shown that an equivalent condition for the strong interference condition (48) and (49) is

$$
h_{12}^{2} \geq h_{11}^{2}+h_{1 R}^{2} \quad h_{21}^{2} \geq h_{22}^{2}+h_{2 R}^{2},
$$

i.e., the strength of the interference link is greater than the sum of the direct link and the corresponding source-relay link.

\section{CONCLUSION}

In this work, we have established the merits of generalized compress-and-forward (CF) type relaying when the relay is oblivious. We have focused on primitive multi-user networks with an oblivious relay where the relay-destination links are out-of-band with finite capacity. We have established the capacity region of the primitive multiple access channel (PMARC) with an oblivious relay by deriving new outerbounds and using the generalized $\mathrm{CF}(\mathrm{GCF})$ relaying scheme for achievability. Additionally, for the primitive interference relay channel with an oblivious relay, we have established a strong interference condition, under which the GCF scheme achieves capacity region. The results obtained in this paper can provide insights towards designing optimal relaying strategies for practical wireless networks, where the codebook information is not present at the relay nodes.

\section{REFERENCES}

[1] T. Cover and A. El Gamal, "Capacity theorems for the relay channel," IEEE Transactions on Information Theory, vol. IT-25, pp. 572-584, 1979.

[2] Y. H. Kim, "Capacity of a class of deterministic relay channels," IEEE Transactions on Information Theory, vol. 54, no. 3, March 2008.

[3] L. Sankaranarayanan and N. B. M. G. Kramer, "On the capacity of the interference channel with a relay," in Proceedings of IEEE International Symposium on Information Theory, July 2008.

[4] O. Sahin and E. Erkip, "Achievable rates for the Gaussian interference relay channel," in Proceedings of the IEEE Global Telecommunications Conference, November 2007.

[5] I. Maric, R. Dabora, and A. Goldsmith, "An outer bound for the Gaussian interfrence channel with a relay," in Proceedings of IEEE Information Theory Workshop, October 2009.

[6] Y. Tian and A. Yener, "The Gaussian interference relay channel: Improved achievable rates and sum rate upperbounds using a potent relay," IEEE Transactions on Information Theory, Special Issue on Interference Networks, vol. 57, no. 5, pp. 2865-2879, May 2011.

[7] A. Sanderovich, S. Shamai, Y. Steinberg, and G. Kramer, "Communication via decentralized processing," IEEE Transactions on Information Theory, vol. 54, no. 7, pp. 3008-3023, July 2008.

[8] O. Simeone, E. Erkip, and S. Shamai, "On codebook information for interference relay channels with out-of-band relaying," IEEE Transactions on Information Theory, Special Issue on Interference Networks, vol. 57, no. 5, pp. 2880-2888, May 2011.

[9] A. E. Gamal and Y.-H. Kim, "Lecture notes in network information theory," available at arXiv:1001.3404v3, March 2010.

[10] Y. Tian and A. Yener, "Relaying for multiuser networks in the absence of codebook information," submitted to IEEE Transactions on Information Theory, November 2011.

[11] X. Wu and L. Xie, "On the optimality of successive decoding in compress-and-forward relay schemes," in Proceedings of 48th Annual Allerton Conference on Communication, Control, and Computing, October 2010.

[12] I. Maric, R. D. Yates, and G. Kramer, "Capacity of interference channels with partial transmitter cooperation," IEEE Transactions on Information Theory, vol. 53, no. 10, pp. 3536-3548, October 2007. 